Deliverable for the Contract with George Mason University

A Prototypical Ontology-supported Intelligent Geospatial Feature Discovery System (iGFDS) for Proliferation Detection

\title{
Nuclear Power Plant Ontology in OWL Format
}

\author{
Prepared by Richard Ward and Alex Sorokine
}

Computational Sciences and Engineering Division

Oak Ridge National Laboratory

Oak Ridge, Tennessee 37831

for

Peng Yue and Liping Di

Center for Spatial Information Science and Systems

George Mason University

4400 University Drive, MSN 6E1

Fairfax, VA 22030-4444

Prepared by

OAK RIDGE NATIONAL LABORATORY

Oak Ridge, Tennessee 37831-6006

managed by UT-BATTELLE, LLC

for the U.S. DEPARTMENT OF ENERGY

under contract DE-AC05-00OR22725 


\section{Nuclear Power Plant Ontology in OWL Format}

This document describes an ontology for Nuclear Power Plant sites for use in software developed by Peng Yue of George Mason University, the intelligent Geospatial Feature Discovery System (iGFDS). The ontology was developed from unclassified and open sources available on the Web, primarily from Wikipedia.

Figures 1 and 2 are screenshots of the Protégé application showing the classes of the Nuclear Power Plant Ontology which was developed in the OWL format.

The ontology contains supplemental information that is not shown in these screenshots and that appears as "comments" in the Annotations window. For example, "SteamGenerator" and "SecondaryCoolant" only apply to "PWR" reactors.

The ontology is based on general professional knowledge of Nuclear Power Plant operation and contains a number of components necessary for understanding and modeling the functionality of a Nuclear Power Plant. The content of the ontology is not limited to equipment and buildings that can be typically observed in the imagery. The things that can be seen are often the higher level items (CoolingTower, TurbineBuilding, ContainmentBuilding, etc.). It should be pointed out that PWR plants will be much easier to identify, generally, compared to BWR plants, due to the characteristic containment structure.

The information on the TransformerYard was obtained from other sources not associated specifically with Nuclear Power Plants

This ontology has to be viewed as a starting point of development ontologies for assisting with identification of Nuclear Power Plant sites with the GMU software. It can be a model for ontologies for other industrial sites. The classes and relations in the ontology have to be rearranged in a way that is most suitable for feature detection. Also the classes pertaining to visible components or signs of operation of a nuclear power plant will have to be linked to classes and properties of the objects in imagery that can be detected by image processing algorithms.

Note that the current ontology does not contain statements about anything that is not present at a Nuclear Power Plant site, for example, "a Nuclear Power Plant DOES NOT HAVE a large pile of coal or other raw materials", which would help logically to differentiate it from other types of power plants. 
References:

1. Reactor Concepts Manual, Boiling Water Reactor Systems, USNRC Technical Training Center, http://www.nrc.gov/reading-rm/basic-ref/teachers/03.pdf

2. The Virtual Nuclear Tourist, BWR Plant Buildings, http://www.nucleartourist.com/areas/areas2.htm

3. Wikipedia, Boiling Water Reactor, http://en.wikipedia.org/wiki/Boiling water reactor.

4. Wikipedia, Pressurized Water Reactor, http://en.wikipedia.org/wiki/Pressurized water reactor

5. Wikipedia, Electrical Substation, http://en.wikipedia.org/wiki/Electrical substation

6. Wikipedia, Heavy Water Reactor, http://en.wikipedia.org/wiki/Heavy water reactor

7. Wikipedia, CANDU Reactor, http://en.wikipedia.org/wiki/CANDU reactor

Delivered with this document: nuclear_power_plant.owl 


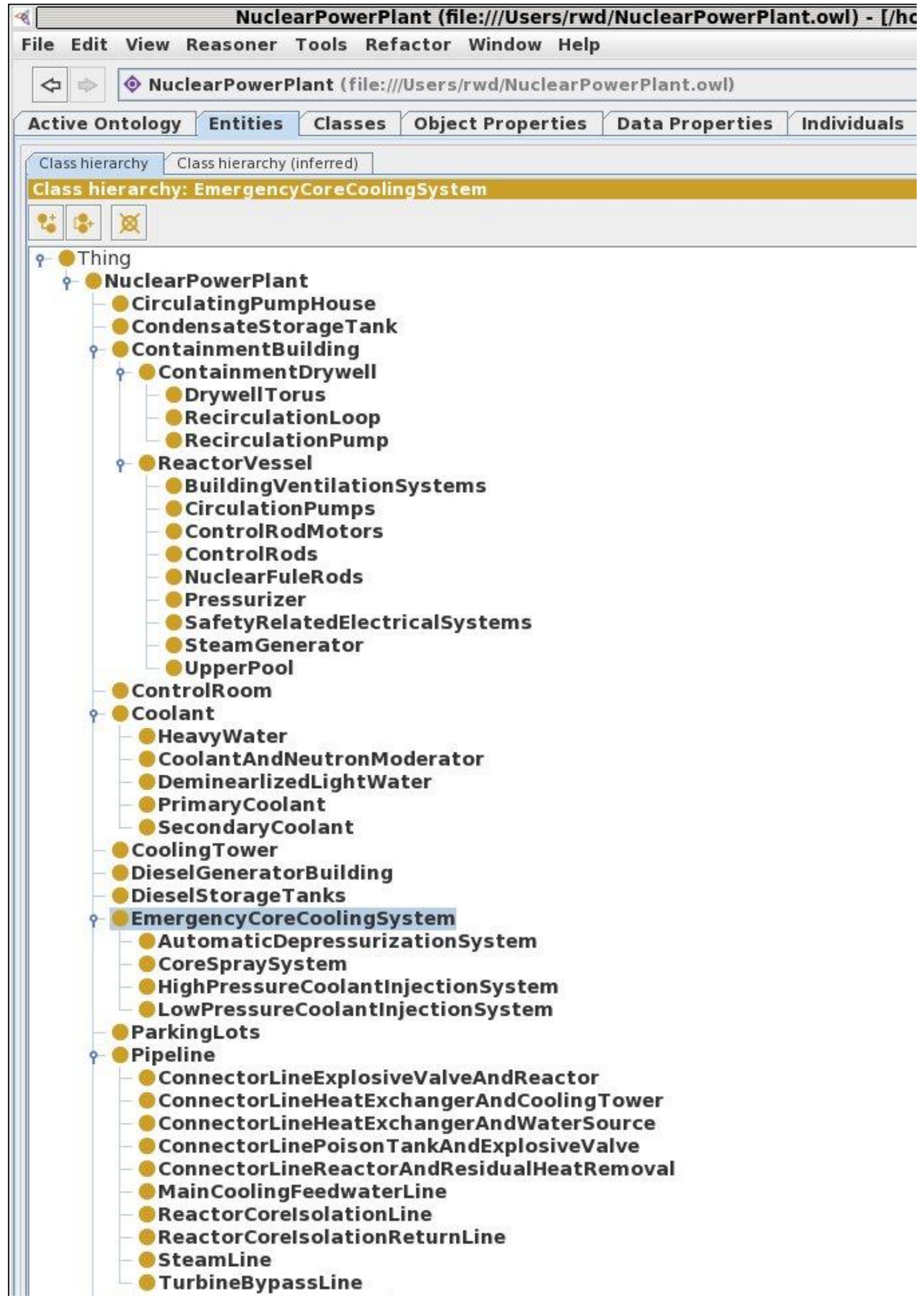

Figure 1. Screenshot of the Nuclear Power Plant Ontology in Protégé 4.1 (first portion). 


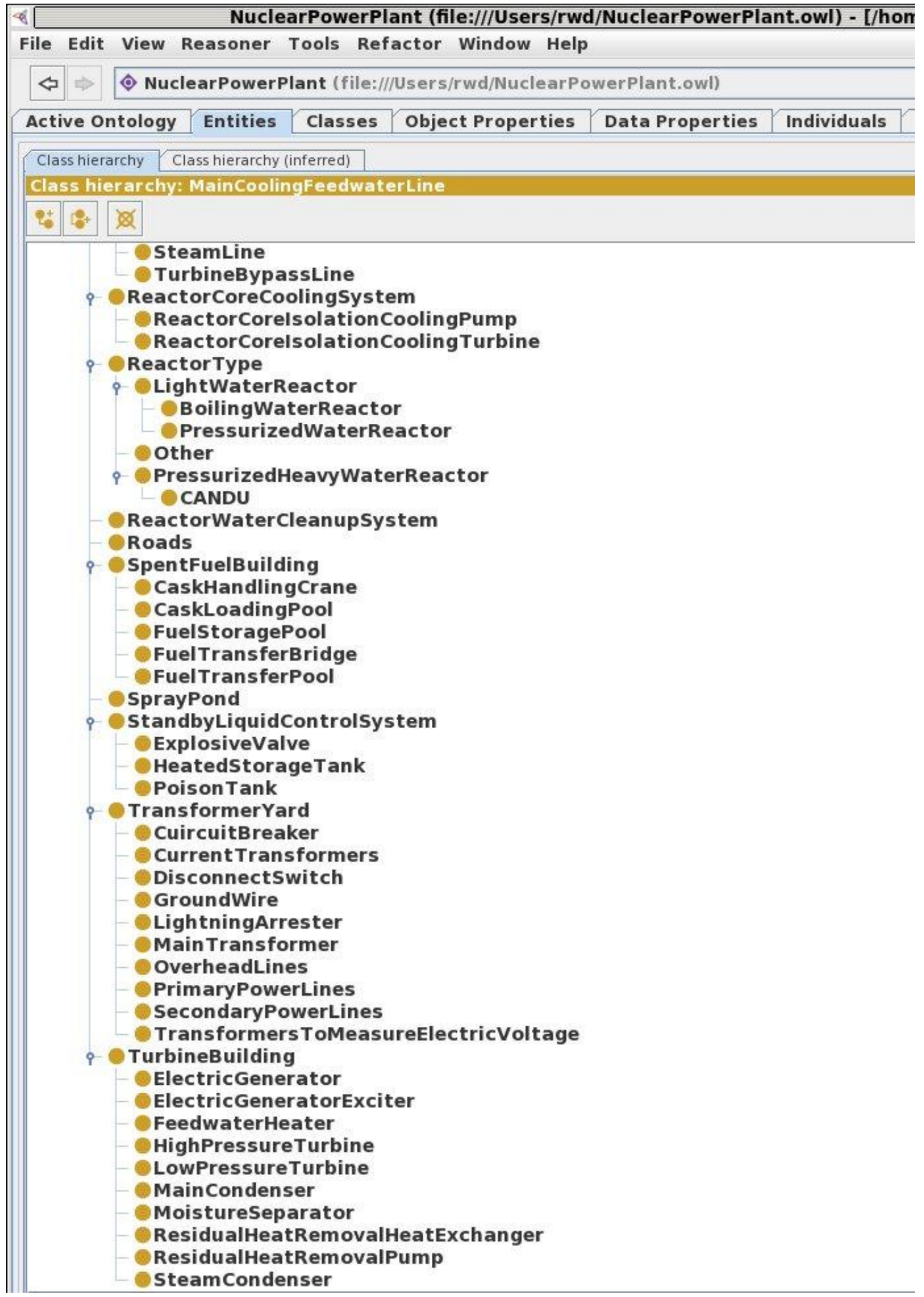

Figure 2. Screenshot of the Nuclear Power Plant Ontology in Protégé 4.1 (second portion). 